\title{
Study on the Sensitivity of high-energy GRB detection using the single-particle technique at an altitude $5200 \mathrm{~m}$ a.s.I
}

\author{
M.Y. Liu ${ }^{* 1,2 \dagger}$ Y.Q. Guo ${ }^{3}$, Danzengluobu ${ }^{1,2}$, C. Liu ${ }^{3}$, T.L. Chen ${ }^{1,2}$, H. Cai ${ }^{3}$, H.J.Li ${ }^{1,2}$ \\ Y.Zhou ${ }^{1}$ W.H. Lei ${ }^{1,2}$, \\ ${ }^{1}$ Physics Department of Science School, Tibet University, Lhasa 850000, Tibet, China \\ ${ }^{2}$ The Key Laboratory of Cosmic Rays, Ministry of Education, Lhasa 850000, Tibet, China \\ ${ }^{3}$ Key Laboratory of Particle Astrophysics, Institute of High Energy Physics, Chinese Academy of \\ Sciences, Beijing 100049, China \\ E-mail: liumaoyuane163.com
}

\begin{abstract}
Owing to the advantages in the wide field of view, high duty cycle and large effective area, ground based high altitude EAS experiments play an important role in studying the high energy gamma ray bursts (GRBs). While shower mode technique provides the most sensitive way in searching for GRBs above $100 \mathrm{GeV}$ energy, the single-particle technique can extend the GRBs search energy down to GeV level.Therefore in this paper,we study the sensitivity of GRB detection using the single-particle technique. by assuming a prototype setup composing three Water Cherenkov Detector (WCD) units locating at 5200m a.s.l. , in PuMa JiangTang, Tibet, China,
\end{abstract}

The 34th International Cosmic Ray Conference,

30 July- 6 August, 2015

The Hague, The Netherlands

\footnotetext{
* Speaker.

${ }^{\dagger}$ The work is funded by the NSF of China (No.11463004,11165013), Tibet University young teachers Scheme,the Natural Science Foundation of China Tibet, The Research project of Chinese Ministry of Education(No. 213036A)
} 


\section{Introduction}

Observations of gamma rays bursts (GRBs) above $10 \mathrm{GeV}$ are important in the research of radiation process and quantum gravitational effects of high-energy GRBs. The space-borne detector of Fermi-LAT has observed tens of GRBs in the energy range from MeV to GeV[1]. However, only a few of photons with energy greater than $10 \mathrm{GeV}$ were detected due to the limitations of the spacebased detector. Extensive air shower (EAS) arrays has its advantages such as a large effective area, high duty cycle, and the wide field of view[2]. However, the thresholds of EAS arrays in operation are larger than hundreds of GeVs. Because the shower development induced by gamma rays below $50 \mathrm{GeV}$ is approaching the end at an altitude of 4,000 meters. The elevation of the present large EAS arrays are all lower than 4300 meters, which results in the poor efficiency of EAS arrays in observation of the tens of $\mathrm{GeV}$-bursts.

A classical method to use is called :single particle technique (SPT). When high energy photons from a GRB reach the atmosphere, they produce cosmic ray cascades that can be detected. The energies are not enough to produce a shower detectable at ground level (even at high altitudes). However, many photons are expected to arrive during the burst, in a short period of time. One would therefore see an increase of the background rate on all the detectors on this time scale. This technique has already been applied in INCA[3] in Bolivia ,ARGO[4] in Tibet and LAGO in South America [5]. The main advantage of single particle technique using water Cherenkov is their sensitivity to photons, which represent up to $90 \%$ of the secondary particles at ground level for high energy photon initiated showers.

Taking all the above considerations into account, we aim to set up a water Cherenkov detector array, which is based on dectection of GRBs using the single particles techniques in a above 5000meter-high region located in PuMa JiangTang, Tibet, China[6][7] . In this paper, we present the simulation result for 3 tanks.

\section{The Simulation of Detector}

The modeling is performed in two steps. Firstly, simulations of cosmic ray interactions with the atmospheric layer were conducted. There was performed for the air shower development in the atmosphere by Corsika (version 6.502). The electromagnetic interactions were described by the EGS4 package, while the hadronic interactions above $80 \mathrm{GeV}$ were reproduced by the QGSJETII.03 model. The low-energy hadronic interactions were described by the GHEISHA package. The altitude of the experimental site was set at $5200 \mathrm{~m}$ above sea level.Primary cosmic ray species, gamma-rays, were assumed to follow a power-law energy spectrum proportional to $\mathrm{E}^{-} 2$ ranging from 1 to $10 \mathrm{GeV}$, and it was thrown from the celestial region with $\theta \leq 40^{\circ}$ and $-180^{\circ} \leq \phi \leq 180^{\circ}$, where $\theta$ denotes the zenith angle and $\phi$ the azimuth angle. The secondary particles were tracked down to a cut-off energy of $1 \mathrm{MeV}$ (electromagnetic component) and $100 \mathrm{MeV}$ (muons and hadrons). Secondly, the secondary particles in the detector were simulated using the Geant 4 package . In the simulations, the altitude of the experimental site is set at $5200 \mathrm{~m}$ above sea level. The Water Cherenkov Detector array is assumed to consist of 3 tanks, each of them is $7.2 \mathrm{~m}$ of diameter and $1 \sim$ $5 \mathrm{~m}$ deep . The calibration of the detector was performed using a test beam. The experimental conditions (trigger logic, time resolution, noise, etc.) were considered via a GEANT4-based code. In 


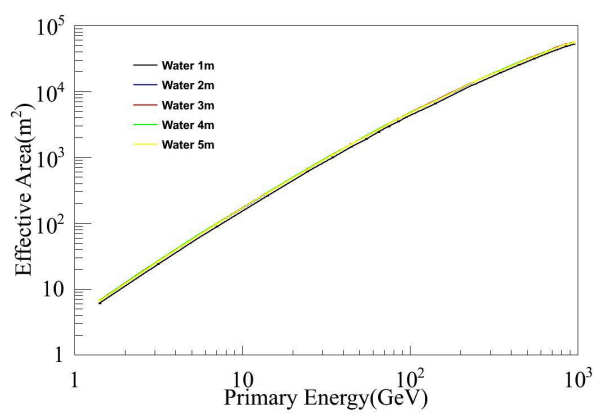

Figure 1: The difference of the effective area with different water depth.

the GEANT4-based detector simulation, the background noise rate of one PMT at the elevation of $5200 \mathrm{~m}$ was $3 \mathrm{kHz} / \mathrm{m}^{2}$, according to the LAGO experiment.

Figure. 1 shows the effective area at different water depths. The effect of water depth on the effective area is small from $1 \mathrm{~m}$ to $5 \mathrm{~m}$. From the economic perspective, a water depth of $1 \mathrm{~m}$ is sufficient. moreover, at $10 \mathrm{GeV}$, the effective area of the array is $200 \mathrm{~m}^{2}$, increasing rapidly to $1,000 \mathrm{~m}^{2}$ at $35 \mathrm{GeV}$ and $5,000 \mathrm{~m}^{2}$ at $100 \mathrm{GeV}$. We conservatively choose a depth of $200 \mathrm{~cm}$ in the further construction of the detector unit.

\section{GRB observation sentitivity}

The GRB observation by Fermi-LAT has initiated a new era of studying on the mechanism$\mathrm{s}$ of high energy emission. Until now, tens of GeV GRBs were observed and five of them with more than $10 \mathrm{GeV}$ photon. Particularly, a nearby super-luminous burst GRB130427A was simultaneously detected by six gamma ray space telescopes(Swift, Fermi-GBM/LAT, Konus-Wind, SPI-ACS/INTEGRAL, AGILE and RHESSI). More excited is that the highest energy LAT photon is greater than $90 \mathrm{GeV}$.

In the present work, two typical GRBs were used, namely, GEB090510 and GRB090902B, to study the sensitivity of our detector. The highest-energy photons of these two GRBs detected by Fermi-LAT are 31 and $34 \mathrm{GeV}$. It was determined that the GRB090510 (T90=0.95 s, red shift $=0.906$, peak energy $=1.66 \mathrm{GeV}$ ) is more suitable for the detection of our detector. To estimate the sensitivity of array, we used GRB090510, one of brightest GRBs observed, as an example. We assumed the extension of power-law component measured by Fermi-GBM and Fermi-LAT. Figure 2 shows that the experiment observed enough significance. As shown in Figure.2, different lines represent different energy truncation positions. GRBs were modeled using a simple power law, and the energy absorption model is simple. GRB090510 and GRB090902B are also shown in the figure. Even in a smaller energy truncation at $30 \mathrm{GeV}$, the effective detection of GRBs was achieved. Conclusively, as shown in Figure.3 GRB090510 can observe obvious variations in the shape of Cerenkov signals. This is in contrast to Fermi-LAT, which can only observe one high-energy photon due to its limited area, thus cannot measure shape information.GRB090902B can detect shapes when the energies of the incoming gamma rays are greater than $30 \mathrm{GeV}$, As shown in Figure.3. 


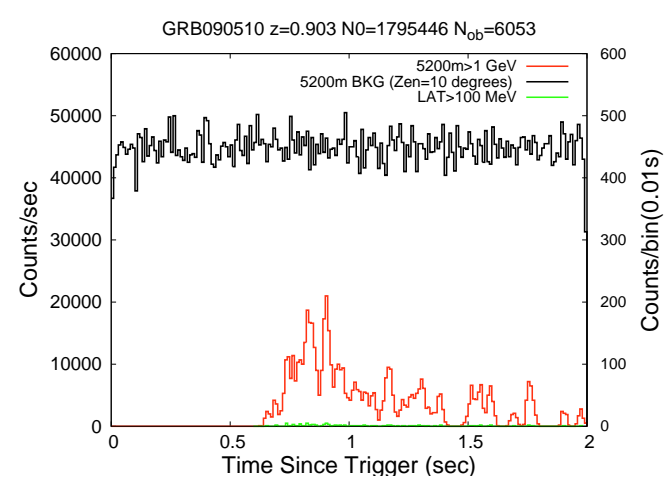

Figure 2: The light change curve of Simulation detection of GRB090510, where the red line is the light change curve observed by the Tibet experiment project when $\geq 1 \mathrm{GeV}$, the green line is the light change curve of Fermi LAT observations, and the black line is the simulation background of Tibet $5200 \mathrm{~m}$.

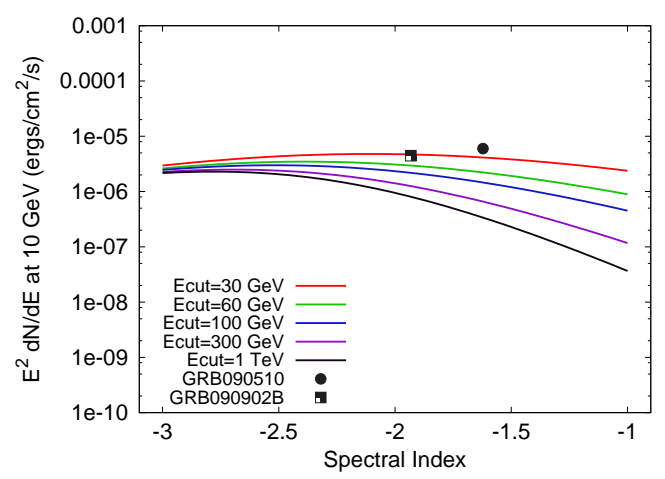

Figure 3: The relations of the gamma ray bursts with energy spectrum sensitivity index and energy truncation position.

\section{Summary}

In this paper we performed the simulation for water cerenkov detector using single particle counting technology in the $5200 \mathrm{~m}$ elevation detection $\mathrm{GeV}$ area GRBs. The by applying the advantages of our experiment is to use few money, to achieve the detection of GRBs, which is regarded to be high performance-price ratio.

\section{References}

[1] The Fermi Gamma-ray Space Telescope,http://fermi.gsfc.nasa.gov/

[2] A.J.Swith,ICRC2008.ID:666.

[3] R. Cabrera et. al, 1999, AS 138, 599.

[4] A. Surdo et. al, ICRC 2003.

[5] D. Allarda et.al,ICRC2009.

[6] Chen Tianlu et.al,ICRC2013.ID:261.

[7] Danzengluobu et al.,ICRC 2011,ID:1348. 